\title{
Noise processes in nanomechanical resonators
}

\author{
A. N. Clelanda) \\ Department of Physics and iQUEST, University of California at Santa Barbara, Santa Barbara, \\ California 93106 \\ M. L. Roukes \\ Department of Physics, California Institute of Technology, Pasadena, California 91125
}

(Received 15 February 2002; accepted for publication 18 June 2002)

\begin{abstract}
Nanomechanical resonators can be fabricated to achieve high natural resonance frequencies, approaching $1 \mathrm{GHz}$, with quality factors in excess of $10^{4}$. These resonators are candidates for use as highly selective rf filters and as precision on-chip clocks. Some fundamental and some nonfundamental noise processes will present limits to the performance of such resonators. These include thermomechanical noise, Nyquist-Johnson noise, and adsorption-desorption noise; other important noise sources include those due to thermal fluctuations and defect motion-induced noise. In this article, we develop a self-contained formalism for treating these noise sources, and use it to estimate the impact that these noise processes will have on the noise of a model nanoscale resonator, consisting of a doubly clamped beam of single-crystal Si with a natural resonance frequency of 1 GHz. (C) 2002 American Institute of Physics. [DOI: 10.1063/1.1499745]
\end{abstract}

\section{INTRODUCTION}

Nanomechanical resonators are rapidly being pushed to smaller size scales and higher operational frequencies, partly due to potential applications as on-chip high- $Q$ filters and clocks. Such resonators would have the potential for replacing bulk quartz crystals and surface-acoustic wave resonators in technological and precision measurement applications, which require extensive separate circuitry and space requirements. High-frequency resonators have been fabricated from bulk $\mathrm{Si}^{1}{ }^{1}$ silicon-on-insulator, ${ }^{2}$ silicon carbide, ${ }^{3}$ silicon nitride, ${ }^{4}$ and from polycrystalline $\mathrm{Si}$ (poly-Si). ${ }^{5,6}$ High resonance frequencies can be achieved using submicron lithography to define doubly clamped beams with relatively large length-to-thickness ratios of $L / t \sim 10-20$. Smaller aspect ratios, with $L / t \sim 2-5$, allow high frequencies to be achieved with less stringent demands on lithographic capability. For these smaller aspect ratios, however, thermoelastic damping begins to become an important source of energy loss and noise, ultimately limiting the quality factor and noise performance. $^{4,7}$

The resonance frequency of a mechanical structure in general scales as $1 / L$, where $L$ is the scale of the resonator. As the size scales are reduced and frequencies increased, however, the short-term stability of the resonator will be limited by certain fundamental noise processes. ${ }^{8}$ These noise processes include the thermomechanical noise generated by the internal loss mechanisms in the resonator, ${ }^{9}$ NyquistJohnson noise from the readout circuitry, ${ }^{10}$ and adsorptiondesorption noise from residual gas molecules in the resonator packaging. ${ }^{11}$ Another noise source is due to temperature fluctuations caused by the finite thermal conductance of the resonator; ${ }^{12}$ these fluctuations are fundamental to any object with finite heat capacity, and are distinct from environmental

\footnotetext{
${ }^{a)}$ Electronic mail: cleland@iquest.ucsb.edu
}

thermal drifts that can be controlled using oven-heated packaging, similar to that used for high-precision quartz clocks. Resonators fabricated from polycrystalline materials, such as those including poly-Si and silicon nitride, are also expected to demonstrate noise from anelastic noise processes caused by grain-boundary and point defect motion. ${ }^{13}$

At present, there does not exist a single self-contained formalism for describing the resonance and noise properties of nanomechanical resonators. In the first part of this work, we therefore develop such a formalism, based on the wellknown Euler-Bernoulli theory of beams. We hope that this will provide a clear and useful framework for future developments in the field. In the latter part of the work, we use this formalism to calculate the effects of the most significant and fundamental, classical sources of noise on resonator performance. The importance of thermomechanical noise, arising from the nonzero dissipation and temperature of a resonator, has been recognized for some time, and its effects have been included in previous noise analyses of mechanical resonators. ${ }^{9} 14$ Other noise sources have also been included in more recent analyses, as mentioned herein. However, our results are not in agreement with the results of these more recent works, in particular, in terms of the magnitude of the impact of the noise, as well as the method of analysis of some of the noise sources, in particular, that of the effect of temperature fluctuations. We have also included a discussion of defect noise, that to our knowledge has not previously been considered.

We do not consider noise or physical limitations produced by particular transducer implementations. Electrostatically driven and detected resonators suffer from surface charge motion; magnetomotive approaches require large stable ambient magnetic fields; optical approaches require stable sources of monochromatic light. We are more concerned with the limitations set by the physics of resonator 


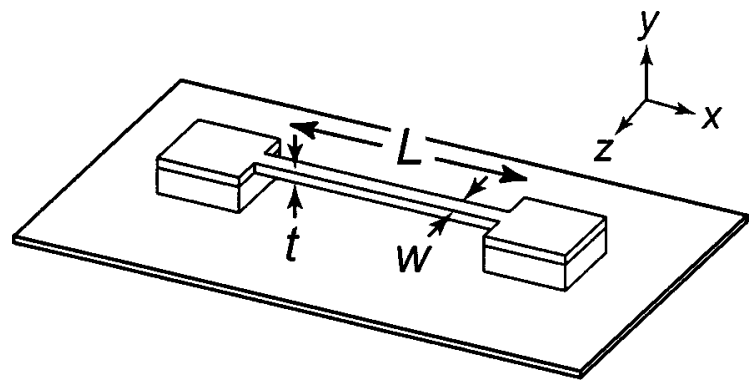

FIG. 1. Doubly clamped beam with length $L$, width $w$ and thickness $t$. The end supports are assumed infinitely rigid.

behavior, and transducer approaches should be evaluated separately from these.

\section{DOUBLY CLAMPED BEAM RESONATORS}

\section{A. Euler-Bernoulli theory}

In Fig. 1, we show the structure forming the basis of our calculations: A doubly clamped beam of length $L$, width $w$, and thickness $t$, oriented along the $x$ axis, driven into flexural resonance with displacement along the $y$ axis.

The dynamic behavior of a flexural beam is most easily treated using the Euler-Bernoulli theory, which applies to beams with aspect ratios $L / t \gg 1 .^{15}$ For an isotropic material, the transverse displacement $Y(x, t)$ of the beam centerline (along the $y$ direction), obeys the differential equation

$$
\rho A \frac{\partial^{2} Y}{\partial t^{2}}(x, t)=-\frac{\partial^{2}}{\partial x^{2}} E I \frac{\partial^{2} Y}{\partial x^{2}}(x, t),
$$

where $\rho$ is the material density, $A=w t$ is the cross-sectional area, $E$ is Young's modulus, and $I=w t^{3} / 12$ is the bending moment of inertia. The clamped ends, at $x=0$ and $x=L$, impose the boundary conditions $Y(0)=Y(L)=0$ and $Y^{\prime}(0)=Y^{\prime}(L)=0$. The solutions have the form

$$
\begin{aligned}
Y_{n}(x, t)= & \left(C_{1 n}\left(\cos k_{n} x-\cosh k_{n} x\right)\right. \\
& \left.+C_{2 n}\left(\sin k_{n}-\sinh k_{n} x\right)\right) \exp \left(-i \Omega_{n} t\right),
\end{aligned}
$$

with eigenvectors $k_{n}$ satisfying $\cos k_{n} L \cosh k_{n} L=1$. The first four eigenvectors are given by $k_{n} L=4.73004,7.8532$, 10.9956, and 14.1372. The angular frequencies $\Omega_{n}$ are given by

$$
\Omega_{n}=\sqrt{\frac{E I}{\rho A}} k_{n}^{2} .
$$

The fundamental eigenfrequency is given by

\begin{tabular}{|c|c|c|c|c|c|}
\hline $\begin{array}{c}L \\
(\mu \mathrm{m})\end{array}$ & $\begin{array}{c}t \\
(\mu \mathrm{m})\end{array}$ & $\begin{array}{c}w \\
(\mu \mathrm{m})\end{array}$ & $\begin{array}{c}M \\
(\mathrm{fg})\end{array}$ & $\begin{array}{c}\nu_{1} \\
(\mathrm{GHz})\end{array}$ & $\begin{array}{c}k_{1} \\
\left(\mu \mathrm{m}^{-1}\right)\end{array}$ \\
\hline 0.66 & 0.05 & 0.05 & 3.84 & 1.00 & 7.17 \\
\hline
\end{tabular}

TABLE I. Numerical solutions for a doubly clamped beam.

\begin{tabular}{lcccc}
\hline \hline & $n=1$ & 2 & 3 & 4 \\
\hline$k_{n} L$ & 4.73004 & 7.8532 & 10.9956 & 14.1372 \\
$\nu_{n} / \nu_{1}$ & 1 & 2.756 & 5.404 & 8.933 \\
$C_{1 n} / L$ & -1.0000 & -1.0000 & -0.9988 & -1.0000 \\
$C_{2 n} / L$ & 0.9825 & 1.0008 & 0.9988 & 1.0000 \\
\hline \hline
\end{tabular}

TABLE II. Parameters for the beam in this calculation.

and the higher modes are $\nu_{n} / \nu_{1}=2.756,5.404$, and 8.933 for $n=2,3$, and 4 .

The eigenfunctions $Y_{n}$ in Eq. (2) are mutually orthogonal, and we normalize them to the beam length, so that

$$
\int_{0}^{L} Y_{n}(x) Y_{m}(x) d x=L^{3} \delta_{m n} .
$$

The corresponding coefficients $C_{1 n}$ and $C_{2 n}$ are listed in Table I. An arbitrary solution $Y(x, t)$ to undriven or driven motion can be written

$$
Y(x, t)=\sum_{n=1}^{\infty} a_{n}(t) Y_{n}(x),
$$

where the amplitudes $a_{n}$ are dimensionless.

The fundamental frequency $\nu_{1}$ is a function of the material parameters $E$ and $\rho$ as well as the beam dimensions $t$ and $L$. High frequencies can be achieved by reducing the overall resonator scale, by choosing stiffer and lighter materials, and by reducing the aspect ratio $L / t$; all three of these approaches are being used, and at present the highest reported frequency is $0.63 \mathrm{GHz}$, for a $\mathrm{SiC}$ beam. ${ }^{16}$ For the purposes of this article, we will focus on a single-crystal Si beam with dimensions as given in Table II; the relevant physical properties for Si are given in Table III, all at room temperature. Our calculations are for the fundamental resonance $n=1$.

In the next section, we discuss the anelastic processes that result in a finite quality factor $Q$ for the beam resonance, described within the context of Zener's model for anelastic solids. In that section, we will describe how the Zener model is included in the formalism described so far. In later sections, we discuss other types of noise that are not described within the context of the Zener model; these have to do with parametric changes in the physical properties of the resonator, such as its mass and length, which cause the natural resonance frequency of the resonator to change, but do not necessarily involve energy dissipation. Any single parametric change can be associated with a change in the resonator

TABLE III. Properties for Si at room temperature.

\begin{tabular}{lcc}
\hline \hline Density & $\rho$ & $2330 \mathrm{~kg} / \mathrm{m}^{3}$ \\
Young's modulus & $E$ & $1.69 \times 10^{11} \mathrm{~N} / \mathrm{m}^{2}$ \\
Thermal conductivity & $\kappa$ & $1.48 \mathrm{~W} / \mathrm{cm} \mathrm{K}^{3}$ \\
Specific heat & $C_{V}$ & $1.64 \mathrm{~J} / \mathrm{cm}^{3} \mathrm{~K}$ \\
Sound speed & $c_{s}$ & $5860 \mathrm{~m} / \mathrm{s}$ \\
Phonon mean-free path & $\alpha_{T}$ & $50 \mathrm{~nm}$ \\
Thermal expansion & $\alpha_{T}$ & $2.8 \times 10^{-6} / \mathrm{K}$ \\
\hline \hline
\end{tabular}


energy, depending on where in the oscillation cycle the change occurs, but given events that occur randomly over the oscillation period, on average, the energy change is zero. The noise sources we discuss include adsorption-desorption noise due to molecules around the resonator, temperature fluctuations that change the length and longitudinal stress in the resonator, and defect motion within the resonator. The latter can to some extent be included in the Zener model, but some modes of defect motion will not generate intrinsic dissipation but instead give rise to parametric changes.

For these sources of parametric noise, an instantaneous measurement of the response of a resonator, as a function of frequency, would indicate the actual dissipation associated with material losses, while a measurement that takes a nonzero time to complete allows the resonator frequency to fluctuate over the period of the measurement, and would give a response curve that appears to be associated with a higher rate of dissipation than is actually present, due to the spread of resonance frequencies that appear over the course of the measurement. Separating these two effects experimentally is a very challenging but intriguing problem.

\section{B. Dissipation in mechanical resonators}

The most significant mechanism for energy loss in a nanomechanical resonator is through intrinsic losses in the beam material, which can be treated using Zener's model for anelastic solids. ${ }^{13}$ Other important loss terms include thermoelastic processes, ${ }^{7}$ which are negligible for the resonator geometry and dimensions given here, and through the transduction mechanism, ${ }^{17}$ which can usually be minimized through design considerations.

In Zener's model, the Hooke's stress-strain relation $\sigma$ $=E \epsilon$, relating the stress $\sigma$ to the strain $\epsilon$, is generalized to allow for mechanical relaxation in the solid:

$$
\sigma+\tau_{\epsilon} \frac{d \sigma}{d t}=E_{R}\left(\epsilon+\tau_{\sigma} \frac{d \epsilon}{d t}\right),
$$

where $E_{R}$ is the relaxed value of Young's modulus. Loads applied slowly generate responses with the relaxed modulus, while rapidly varying loads involve a different value for the modulus.

We consider harmonic stress and strain variations, $\sigma(t)$ $=\sigma e^{i \omega t}$ and $\epsilon(t)=\epsilon e^{i \omega t}$. At low frequencies $\omega \tau \ll 1$, this becomes the standard Hooke's law relation with $E=E_{R}$. At high frequencies $\omega \tau \gg 1$, the modulus becomes $E=E_{U}$ $=\left(\tau_{\sigma} / \tau_{\epsilon}\right) E_{R}$, the unrelaxed Young's modulus. For intermediate frequencies, Young's modulus is complex, of the form

$$
E=E_{\mathrm{eff}}(\omega)\left(1+\frac{i \omega \tau}{1+\omega^{2} \tau^{2}} \Delta\right),
$$

with mean relaxation time $\tau=\left(\tau_{\sigma} \tau_{\epsilon}\right)^{1 / 2}$, fractional modulus difference $\Delta=\left(E_{U}-E_{R}\right) / E_{R}$, and effective Young's modulus

$$
E_{\mathrm{eff}}=\frac{1+\omega^{2} \tau^{2}}{1+\omega^{2} \tau_{\epsilon}^{2}} E_{R} .
$$

Equation (8) implies that the stress $\sigma$ will include a component that is $90^{\circ}$ out of phase with the strain $\epsilon$, which causes energy loss at a rate proportional to $\Delta$. For small $\Delta$, we define the quality factor $Q$ as the ratio of the imaginary to the real part of $E$ :

$$
Q^{-1}=\frac{\omega \tau}{1+\omega^{2} \tau^{2}} \Delta .
$$

We then use the effective Young's modulus $E_{\text {eff }}$ in the Euler-Bernoulli formula, Eq. (1) at frequency $\omega$,

$$
\omega^{2} \rho A Y(x)=E_{\mathrm{eff}}(\omega) I\left(1+\frac{i}{Q}\right) \frac{\partial^{4} Y}{\partial x^{4}}(x) .
$$

The spatial solutions $Y(x)$ are the same as for Eq. (1), but the dispersion relation giving the damped eigenfrequencies $\Omega_{n}^{\prime}$ in terms of the undamped frequencies $\Omega_{n}$ is

$$
\Omega_{n}^{\prime}=\left(1+\frac{i}{2 Q}\right) \Omega_{n},
$$

for small dissipation $Q^{-1}$. The imaginary part of $\Omega_{n}^{\prime}$ implies that the $n$th eigenmode will decay in amplitude as $\exp \left(-\Omega_{n} t / 2 Q\right)$.

\section{Driven damped beams}

We now add a harmonic driving force $F(x, t)$ $=f(x) \exp \left(i \omega_{c} t\right)$, where $f(x)$ is the position-dependent force per unit length. The force is uniform across the beam cross section and directed along $y$, and the carrier frequency $\omega_{c}$ is close to $\Omega_{1}$. The equation of motion is ${ }^{15}$

$$
\rho A \frac{\partial^{2} Y}{\partial t^{2}}+E A \frac{\partial^{4} Y}{\partial x^{4}}=f(x) e^{i \omega_{c} t} .
$$

We solve this equation for long times, $\Omega_{1} t / Q \gg 1$, so any transients damp out. The solution then has the form $Y(x, t)$ $=Y(x) e^{i \omega_{c} t}$. The amplitude $Y(x)$ may be complex, so that the motion is not necessarily in phase with the force $F$. Expanding the displacement in terms of the eigenfunctions $Y_{n}$,

$$
-\omega_{c}^{2} \rho A \sum_{n=1}^{\infty} a_{n} Y_{n}(x)+E A \sum_{n=1}^{\infty} a_{n} \frac{\partial^{4} Y_{n}}{\partial x^{4}}=f(x) .
$$

Using the defining relation for the eigenfunctions, Eq. (1), the dispersion relation, Eq. (12), and the orthogonality relations, Eq. (5), this can be written

$$
\left(\Omega_{m}^{\prime 2}-\omega_{c}^{2}\right) a_{m}=\frac{1}{\rho A L^{3}} \int_{0}^{L} Y_{m}(x) f(x) d x,
$$

for each term $m$ in the expansion. For $\omega_{c}$ close to $\Omega_{1}$, only the $m=1$ term in Eq. (15) has a significant amplitude, given by

$$
a_{1}=\frac{1}{\rho A L^{3}} \frac{1}{\Omega_{1}^{2}-\omega_{c}^{2}+i \Omega_{1}^{2} / Q} \int_{0}^{L} Y_{1}(x) f(x) d x,
$$

for small dissipation $Q^{-1}$.

We now take a uniform force, $f(x)=f$. The integral in Eq. (16) is then 


$$
\eta_{1}=\frac{1}{L^{2}} \int_{0}^{L} Y_{1}(x) d x=0.8309 .
$$

The amplitude is then

$$
a_{1}=\frac{\eta_{1}}{\Omega_{1}^{2}-\omega_{c}^{2}+i \Omega_{1}^{2} / Q} \frac{f}{M},
$$

where $M=\rho A L$ is the mass of the beam, and the corresponding displacement of the beam is $Y(x, t)=a_{1} Y_{1}(x) \exp \left(i \omega_{c} t\right)$.

If the force distribution $f(x)$ is instead chosen to be proportional to the eigenfunction $Y_{1}(x)$, the integral Eq. (16) is unity, so that $\eta_{1}$ in Eq. (18) is replaced by the number 1 .

We point out that the response function, Eq. (18), while similar to that of a damped, one-dimensional harmonic oscillator, differs slightly in the $Q$-dependent denominator, but the difference is only apparent for small values of $Q$ : For values of $Q$ greater than 15 , the fractional difference at any frequency is less than $1 \%$.

\section{NOISE IN DRIVEN DAMPED BEAMS}

Systems that dissipate energy are necessarily sources of noise; the converse is also often true. This is the basic statement of the fluctuation-dissipation theorem, and is best known in relation to electrical circuits, where it is termed the Nyquist-Johnson theorem. An electrical circuit element with an electrical impedance $Z(\omega)$ that has a nonzero real part, $R(\omega)=\operatorname{Re} Z(\omega)$, will be a source of noise, that is, of fluctuations in the voltage $V(t)$ across the impedance $Z$, or equivalently in the current $I(t)$ through $Z$. A voltmeter placed across the circuit element will measure an instantaneous voltage that fluctuates with a Gaussian distribution in amplitude, with zero average value, and a width that is determined only by $R(\omega)$ and the temperature $T$. A useful way to quantify the noise is to use the average spectral density of the noise in angular frequency space, defined for a noise voltage $V(t)$ by

$$
S_{V}(\omega)=\left\langle\int_{-\infty}^{\infty} V^{2}(t) \cos (\omega t) d t\right\rangle .
$$

Here the angle brackets $\langle\ldots\rangle$ indicate that a statistical ensemble average, over many equivalent systems, is to be taken. The spectral density is proportional to the electrical noise power in a unit bandwidth. The Nyquist-Johnson theorem states that this quantity is given by $S_{V}(\omega)$ $=(2 / \pi) R(\omega) \hbar \omega \operatorname{coth}\left(\hbar \omega / k_{B} T\right)$. At high temperatures or low frequencies, such that $k_{B} T \gg \hbar \omega$, this approaches the classical limit $S_{V}(\omega) \rightarrow 2 k_{B} T R(\omega) / \pi$. The spectral noise density $S_{V}(f)$, as a function of frequency $f=\omega / 2 \pi$, is given in the high-temperature limit by $S_{V}(f)=2 \pi S_{V}(\omega)=4 k_{B} T R(f)$. The metric units of $S_{V}(f)$ are $\mathrm{V}^{2} / \mathrm{Hz}$. The corresponding current spectral noise density is $S_{I}(f)=S_{V}(f) / R^{2}(f)$ $\rightarrow 4 k_{B} T / R(f)$, in the high temperature limit, with units of $\mathrm{A}^{2} / \mathrm{Hz}$.

The fluctuation-dissipation theorem applies to mechanical resonators with nonzero dissipation, i.e., with finite $Q$, and ensures that the mechanical resonator will also be a source of noise, but due to the resonant nature of the re- sponse of the resonator, the noise spectral density takes on a somewhat different form. We will only treat the hightemperature limit, $k_{B} T \gg \hbar \omega$, for the resonator noise.

\section{A. Dissipation-induced amplitude noise}

The displacement of a forced, damped beam driven near its fundamental frequency is given by Eq. (18). In the absence of noise, this solution represents pure harmonic motion at the carrier frequency $\omega_{c}$. As discussed, the nonzero value of $Q^{-1}$ and temperature $T$ necessitates the presence of noise, from the fluctuation-dissipation theorem. Regardless of the origin of the dissipation mechanism, it acts to thermalize the motion of the resonator, so that in the presence of dissipation only (no driving force), the mean energy $\left\langle E_{n}\right\rangle$ for each mode $n$ of the resonator will be given by $\left\langle E_{n}\right\rangle=k_{B} T$, where $T$ is the physical temperature of the resonator. This noise term has been considered by a number of authors. ${ }^{9,14}$

The thermalization occurs due to the presence of a noise force $f_{N}(x, t)$ per unit length of the beam. Each point on the beam experiences a noise force with the same spectral density, but fluctuating independently from other points; the noise at any two points on the beam is uncorrelated. The noise be written as an expansion in terms of the eigenfunctions $Y_{n}(x)$,

$$
f_{N}(x, t)=\frac{1}{L} \sum_{n=1}^{\infty} f_{N_{n}}(t) Y_{n}(x),
$$

where the force $f_{N_{n}}$ associated with the mode $n$ is uncorrelated with that for other modes $n^{\prime}$; the factor $1 / L$ appears because of the normalization of the $Y_{n}$.

The noise force $f_{N_{n}}(t)$ has a white spectral density $S_{f_{n}}(\omega)$, and a Gaussian distribution with a zero mean. The magnitude of the spectral density $S_{f_{n}}$ may be evaluated by requiring that it achieve thermal equilibrium for each mode $n$. The spectral density of the noise-driven amplitude $a_{n}$ of the $n$th mode is given by

$$
S_{a_{n}}(\omega)=\frac{1}{\left(\Omega_{n}^{2}-\omega^{2}\right)^{2}+\left(\Omega_{n}^{2} / Q\right)^{2}} \frac{S_{f_{n}}(\omega)}{M^{2}} .
$$

The SI units for $S_{f_{n}}$ are $(\mathrm{N} / \mathrm{m})^{2} /(\mathrm{rad} / \mathrm{s})=\mathrm{kg}^{2} /\left(\mathrm{s}^{3} \mathrm{rad}\right)$. Those for $S_{a_{n}}$ are $1 /(\mathrm{rad} / \mathrm{s})$, because $a_{n}$ is dimensionless.

The kinetic energy $K E_{n}$ of the $n$th mode associated with the spectral density $S_{a_{n}}$ is given by

$$
\begin{aligned}
\left\langle K E_{n}\right\rangle & =\frac{1}{2} \int_{0}^{\infty} \int_{0}^{L} \rho A \omega^{2} S_{a_{n}}(\omega) Y_{n}^{2}(x) d x d \omega \\
& =\frac{1}{2} \int_{0}^{\infty} \rho A L^{3} \omega^{2} S_{a_{n}}(\omega) d \omega \approx \frac{\pi}{4} \frac{Q L^{2}}{\Omega_{n}} \frac{S_{f_{n}}(\omega)}{M},
\end{aligned}
$$

where the last equality becomes exact in the limit $Q^{-1} \rightarrow 0$. The error in Eq. (22) for finite $Q$ is less than $1 \%$ for $Q$ $>10$.

In order that this yield thermal equilibrium, the kinetic energy is $\left\langle K E_{n}\right\rangle=\frac{1}{2} k_{B} T$, so the spectral density $S_{f_{n}}$ must be given by 


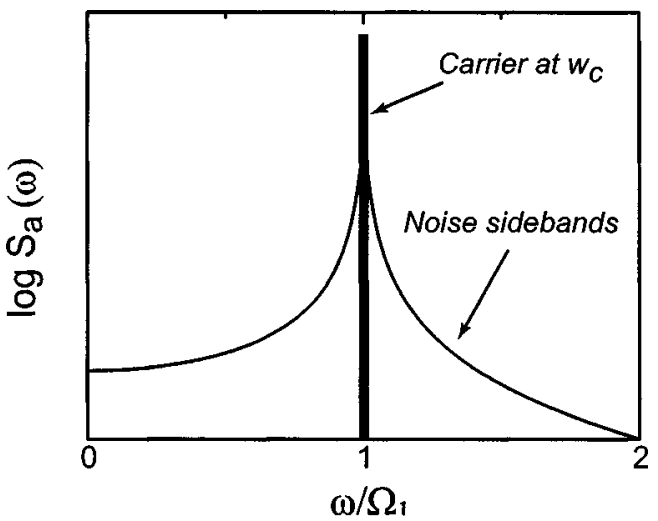

FIG. 2. Frequency spectrum of a driven beam in the presence of noise, showing both the central driven peak as well as the noise sidebands.

$$
S_{f_{n}}(\omega)=\frac{2 k_{B} T M \Omega_{n}}{\pi Q L^{2}} .
$$

The term $L^{2}$ appears in Eq. (23) because $f_{N_{n}}$ is the force per unit length of beam. An equivalent derivation for a onedimensional simple harmonic oscillator yields the force density $S_{F}(\omega)=2 k_{B} T M \Omega / \pi Q$. We can write the spectral density of the thermally driven amplitude as

$$
S_{a_{n}}(\omega)=\frac{\Omega_{n}}{\left(\Omega_{n}^{2}-\omega^{2}\right)^{2}+\left(\Omega_{n}^{2} / Q\right)^{2}} \frac{2 k_{B} T}{\pi M L^{2} Q} .
$$

When superposed with a driving force with a carrier frequency $\omega_{c}=\Omega_{1}$, the amplitude noise power consists of a $\delta$-function peak at the carrier superposed with the Lorentzian given by Eq. (24), as sketched in Fig. 2.

\section{B. Dissipation-induced phase noise}

The form in Eq. (24) represents frequency-distributed amplitude noise. Equivalent expressions can be written for the phase noise, the fractional frequency noise, and the Allan variance, ${ }^{18}$ which are useful for time-keeping and filter applications. We note that the different expressions are all equivalent ways of expressing the same noise, and do not represent additional sources of noise. The resonator is driven by the carrier signal near its resonance frequency $\Omega_{1}$, and in addition by dissipation-induced noise. The time-dependent amplitude is then

$$
a(t)=a_{0} \sin \left(\omega_{c} t+\phi(t)+\theta\right),
$$

where $\phi(t)$ represents a phase variation from the carrier at frequency $\omega_{c} \approx \Omega_{1}$; the amplitude $a_{0}$ is constant, and $\theta$ is a phase offset. Following Robins, ${ }^{19}$ we pick one frequency component at $\omega$ for the phase variation, $\phi(t)=\phi_{0} \sin (\omega t)$. Assuming small maximum deviation $\phi_{0}$, the amplitude may be written

$$
\begin{aligned}
a(t)= & a_{0} \sin \left(\omega_{c} t+\theta\right)+a_{0} \frac{\phi_{0}}{2} \sin \left(\left(\omega_{c}+\omega\right) t\right) \\
& -a_{0} \frac{\phi_{0}}{2} \sin \left(\left(\omega_{c}-\omega\right) t\right) .
\end{aligned}
$$

The phase variation at $\omega$ generates sidebands spaced $\pm \omega$ from the carrier, with amplitude $\pm a_{0} \phi_{0} / 2$. The lower sideband is phase coherent with the upper sideband, with the opposite sign; this is characteristic of phase noise. Independent sideband signals can be generated by adding an amplitude noise source $M(t)$ to the phase noise $\phi(t)$, so that the amplitude is written as

$$
a(t)=a_{0}(1+M(t)) \sin \left(\omega_{c} t+\phi(t)+\theta\right) .
$$

We consider a single component at $\omega$ for both the phase and amplitude modulation, so that

$$
\begin{aligned}
& M(t)=M_{0} \sin (\omega t) \\
& \phi(t)=\phi_{0} \sin (\omega t) .
\end{aligned}
$$

Again assuming small variations, this can be written as

$$
\begin{aligned}
a(t)= & a_{0} \sin \left(\omega_{c} t+\theta\right)+\frac{1}{2} a_{0}\left(M_{0}+\phi_{0}\right) \sin \left(\left(\omega_{c}+\omega\right) t\right) \\
& +\frac{1}{2} a_{0}\left(M_{0}-\phi_{0}\right) \sin \left(\left(\omega_{c}-\omega\right) t\right)
\end{aligned}
$$

Setting the amplitude $M_{0}=\phi_{0}$, the lower sideband disappears and we are left with the independent upper sideband term,

$$
a(t)=a_{0} \sin \left(\omega_{c} t+\theta\right)+a_{0} \phi_{0} \sin \left(\left(\omega_{c}+\omega\right) t\right) .
$$

Choosing the opposite sign relation $M_{0}=-\phi_{0}$ allows the lower sideband to be chosen.

A noise signal at a frequency offset from the carrier can be created from the superposition of a phase and an amplitude modulation of the original carrier. In applications where the resonator is to be used as a frequency source or a clock, the amplitude modulation is unimportant: Use of a zerocrossing detector, or a perfect limiter, eliminates the effects of the amplitude modulation. We therefore ignore this noise source. This is equivalent, from the arguments leading to Eq. (26), to limiting the noise to that which is phase coherent between the upper and lower sidebands, with amplitudes $a\left(\omega_{c}+\omega\right)=-a\left(\omega_{c}-\omega\right)$. Noise which has the opposite phase relation, with $a\left(\omega_{c}+\omega\right)=+a\left(\omega_{c}-\omega\right)$, is due to amplitude modulation. Noise associated with only one sideband consists of the sum or difference of these two "modes".

Dissipation-induced noise, of the form given by Eq. (24), is intrinsically phase incoherent on opposite sides of the carrier signal at $\omega_{c}$. Half of the noise power is therefore associated with amplitude modulation, and half with phase modulation; the phase noise power is therefore half the original total noise power.

We can evaluate the dissipation-induced phase noise for a resonator driven at its fundamental resonance frequency. We drive the resonator with a force $f$ per unit length, at the frequency $\omega_{c}=\Omega_{1}$. The amplitude for the response is given by Eq. (18),

$$
a_{1}=-i \frac{\eta_{1}}{\Omega_{1}^{2}} \frac{Q f}{M} .
$$

The amplitude lags the force by $90^{\circ}$, and includes the multiplicative factor $Q$. Dissipation generates incoherent noise, 
distributed about the carrier with noise power given by Eq. (24). The phase noise power density $S_{\phi}(\omega)$ at frequency $\omega$ from the carrier frequency is then given by

$$
\begin{aligned}
S_{\phi}(\omega) & =\frac{1}{2} \frac{S_{a_{1}}\left(\Omega_{1}+\omega\right)}{\left|a_{1}\right|^{2}} \\
& =\frac{\Omega_{1}}{\left(2 \Omega_{1} \omega+\omega^{2}\right)^{2}+\left(\Omega_{1}^{2} / Q\right)^{2}} \frac{k_{B} T}{\pi\left|a_{1}\right|^{2} L^{2} M Q} .
\end{aligned}
$$

For frequencies that are well off the peak resonance, $\omega$ $\gg \Omega_{1} / Q$, but small compared to the resonance frequency, $\omega \ll \Omega_{1}$, we may approximate the denominator as

$$
\begin{aligned}
S_{\phi}(\omega) & \approx \frac{k_{B} T}{4 \pi \Omega_{1} \omega^{2}\left|a_{1}\right|^{2} L^{2} M Q} \\
& \approx \frac{k_{B} T \Omega_{1}}{8 \pi E_{c} Q \omega^{2}} \quad\left(\Omega_{1} / Q \ll \omega \ll \Omega_{1}\right) .
\end{aligned}
$$

Here, we define the energy $E_{c}$ at the carrier frequency, $E_{c}$ $=M \Omega_{1}^{2} L^{2}\left|a_{1}\right|^{2} / 2$. This can also be written in terms of the power $P_{c}=\Omega_{1} E_{c} / Q$ needed to maintain the carrier amplitude, i.e., that needed to counter the loss due to the nonzero value of $1 / Q$ :

$$
S_{\phi}(\omega) \approx \frac{k_{B} T}{8 \pi P_{c} Q^{2}}\left(\frac{\Omega_{1}}{\omega}\right)^{2} \quad\left(\Omega_{1} / Q \ll \omega \ll \Omega_{1}\right) .
$$

We can also write this expression in terms of frequency $f$ $=2 \pi \omega$,

$$
S_{\phi}(f) \approx \frac{k_{B} T}{4 P_{c} Q^{2}}\left(\frac{\nu_{1}}{f}\right)^{2} \quad\left(\nu_{1} / Q \ll f \ll \nu_{1}\right) .
$$

\section{Frequency noise}

The phase fluctuations can also be viewed as frequency fluctuations, where the amplitude $a(t)$ has a time dependence

$$
a(t)=a_{0} \sin \left(\int_{-\infty}^{t} \omega\left(t^{\prime}\right) d t^{\prime}+\theta\right) .
$$

The time-dependent frequency $\omega(t)$ is related to the carrier frequency $\omega_{c}$ and the phase $\phi(t)$ by

$$
\omega(t)=\frac{d\left(\omega_{c} t+\phi(t)\right)}{d t}=\omega_{c}+\frac{d \phi}{d t} .
$$

We define time-dependent frequency variation $\delta \omega(t)$ as

$$
\delta \omega(t)=\omega(t)-\omega_{c}=\frac{d \phi(t)}{d t} .
$$

We consider a single-phase modulation component, so that $\phi(t)=\phi_{0} \sin (\omega t)$. The frequency variation

$$
\delta \omega(t)=\delta \omega_{0} \cos (\omega t)=\omega \phi_{0} \cos (\omega t)
$$

represents a sinusoidal variation of the frequency, with amplitude $\delta \omega_{0}=\omega \phi_{0}$, modulated at $\omega$. The time-dependent amplitude in Eq. (36) is

$$
\begin{aligned}
a(t)= & a_{0} \sin \left(\omega_{c} t+\left(\delta \omega_{0} / \omega\right) \sin (\omega t)+\theta\right) \\
= & a_{0} \sin \left(\omega_{c} t\right)+\frac{1}{2} a_{0} \frac{\delta \omega_{0}}{\omega} \sin \left(\left(\omega_{c}+\omega\right) t\right) \\
& -\frac{1}{2} a_{0} \frac{\delta \omega_{0}}{\omega} \sin \left(\left(\omega_{c}-\omega\right) t\right),
\end{aligned}
$$

a result very similar to that for phase variations, Eq. (26).

The arguments leading to the spectral density, Eq. (34), may be reworked to yield the equivalent expression for the frequency variation noise density $S_{\delta \omega}$. A more useful quantity is the fractional frequency variation, defined as $y$ $=\delta \omega / \omega_{c}$. The noise density for $y$ is related to that for the phase noise density by

$$
S_{y}(\omega)=\left(\frac{\partial y}{\partial \phi}\right)^{2} S_{\phi}(\omega)=\left(\frac{\omega}{\omega_{c}}\right)^{2} S_{\phi}(\omega),
$$

where we use the fact that modulation at $\omega$ generates sidebands at $\pm \omega$ from the carrier at $\omega_{c}$. From Eq. (34), we then have

$$
S_{y}(\omega) \approx \frac{k_{B} T}{8 \pi P_{c} Q^{2}} \quad\left(\omega_{c} / Q \ll \omega \ll \omega_{c}\right) .
$$

In the frequency domain, this is

$$
S_{y}(f) \approx \frac{\pi k_{B} T}{4 P_{c} Q^{2}} \quad\left(\nu_{c} / Q \ll f \ll \nu_{c}\right) .
$$

\section{Allan variance}

A third useful quantity, commonly used to compare frequency standards, is the Allan variance $\sigma_{A}\left(\tau_{A}\right){ }^{20,21}$ The phase and frequency noise are defined in the frequency domain; the Allan variance is defined in the time domain, as the variance over time in the measured frequency of a source, each measurement averaged over a time interval $\tau_{A}$, with zero-dead time between measurement intervals. The defining expression for the square of the Allan variance is

$$
\sigma_{A}^{2}\left(\tau_{A}\right)=\frac{1}{2 f_{c}^{2}} \frac{1}{N-1} \sum_{m=2}^{N}\left(\bar{f}_{m}-\bar{f}_{m-1}\right)^{2},
$$

where $\bar{f}_{m}$ is the average frequency measured over the $m$ th time interval, of length $\Delta t=\tau_{A}$, and $f_{c}$ is the nominal carrier frequency. The squared Allan variance is related to the phase noise density by ${ }^{21}$

$$
\sigma_{A}^{2}\left(\tau_{A}\right)=2\left(\frac{2}{\omega_{c} \tau_{A}}\right)^{2} \int_{0}^{\infty} S_{\phi}(\omega) \sin ^{4}\left(\omega \tau_{A} / 2\right) d \omega,
$$

where $\omega_{c}=2 \pi f_{c}$ and $\omega$ is the modulation frequency.

The Allan variance can be calculated for various functional forms for the phase noise density. For a fractional frequency noise that has a $1 / f$ component, so that $S_{y}(\omega)$ $=A\left(\omega_{c} / \omega\right)$, where $A$ is a scale factor, the phase noise density is $S_{\phi}(\omega)=A\left(\omega_{c} / \omega\right)^{3}$, and the Allan variance is

$$
\sigma_{A}\left(\tau_{A}\right)=\sqrt{2 \log _{e} 2 A \omega_{c}} .
$$




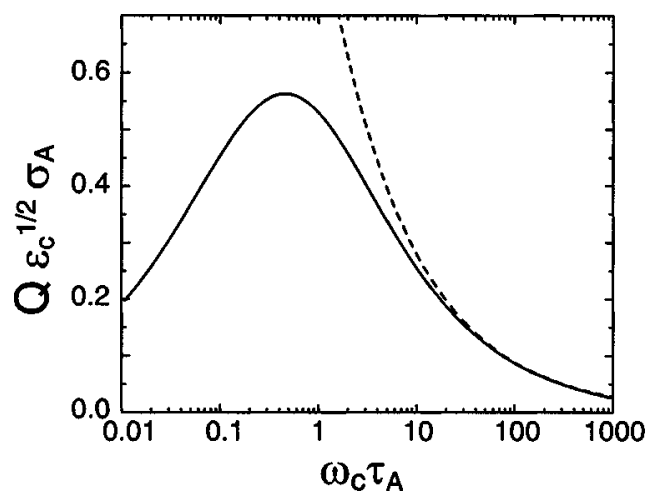

FIG. 3. Dependence of the Allan variance $\sigma_{A}$ on the dimensionless time interval $\omega_{c} \tau$; the Allan variance has been scaled to remove the overall dependence on $Q$ and on drive energy $\varepsilon_{c}$. The full dependence [from Eq. (32)] is plotted as a solid line, while the approximate form [Eq. (50)] is plotted as a dotted line.

Hence a $1 / f$ fractional frequency noise yields an Allan variance that is independent of the measurement time interval.

For a source that displays frequency drift, the fractional frequency noise will have the form $S_{y}(\omega)=B\left(\omega_{c} / \omega\right)^{2}$; the phase noise density is then $S_{\phi}(\omega)=B\left(\omega_{c} / \omega\right)^{4}$, and the Allan variance is

$$
\sigma_{A}\left(\tau_{A}\right)=\sqrt{\frac{\pi}{3} B \omega_{c}^{2} \tau_{A}} .
$$

Finally, for a white fractional frequency noise density $S_{y}(\omega)=C$, the phase noise density has the form $S_{\phi}(\omega)$ $=C\left(\omega_{c} / \omega\right)^{2}$, and the Allan variance is

$$
\sigma_{A}\left(\tau_{A}\right)=\sqrt{\frac{\pi C}{\tau_{A}}} .
$$

In particular, for the approximate form for the phase noise density Eq. (34), the Allan variance is

$$
\sigma_{A}\left(\tau_{A}\right)=\sqrt{\frac{k_{B} T}{8 P_{c} Q^{2} \tau_{A}}} .
$$

Defining the dimensionless drive energy $\varepsilon_{c}$ as the ratio of drive energy per cycle to the thermal energy, $\varepsilon_{c}$ $=2 \pi P_{c} / \omega_{c} k_{B} T$, we have

$$
\sigma_{A}\left(\tau_{A}\right)=\frac{1}{Q} \sqrt{\frac{\pi}{4 \varepsilon_{c} \omega_{c} \tau_{A}}} .
$$

We see that the Allan variance falls inversely with the square root of the product $\omega_{c} \tau_{A}$, and it is also proportional to the dissipation $Q^{-1}$. Other things being equal, increasing the resonator frequency $\omega_{c}$ lowers the Allan variance.

In Fig. 3, we display the approximate result Eq. (50) as a function of $\omega_{c} \tau_{A}$, scaled to remove the dependence on $Q$ and on $\varepsilon_{c}$; we also show the full result obtained from integrating Eq. (32), for values of $Q>100$; for values of $Q$ less than this, the calculated value for the scaled variance falls below that plotted.

We see that the approximate expression given by Eq. (50) works quite well for averaging times $\tau_{A}$ more than a few tens of the oscillation period $2 \pi / \omega_{c}$; in time-keeping or

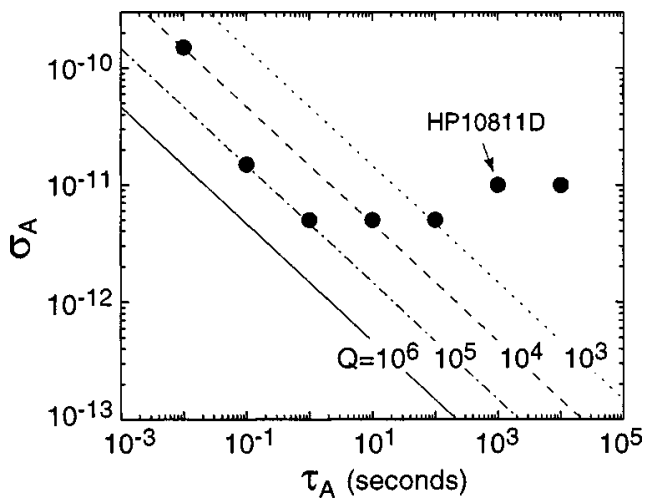

FIG. 4. Allan variance $\sigma_{A}$ as a function of measurement interval $\tau_{A}$, calculated for the model $1 \mathrm{GHz}$ resonator for a range of values of $Q$ from $10^{3}$ to $10^{6}$. The drive power level $P_{c}$ was chosen so that the amplitude of motion $a_{1}=t$, the beam thickness, For comparison, we also display the variance for a $10 \mathrm{MHz}$ quartz crystal frequency standard, the HP10811D; figures taken from manufacturer's specifications.

frequency-lock applications, one is typically interested in times much longer than this, so the approximate form is quite adequate.

We now turn to examining what the implications are for the fundamental sources of noise in our model resonator. We will focus on calculating the predicted dependence of the Allan variance.

\section{E. Model resonator $Q$-dependent noise}

We calculate the dependence of the Allan variance $\sigma_{A}$ on the time interval and $Q$ for our model $1 \mathrm{GHz}$ resonator, described in Table II. The results of the calculation are shown in Fig. 4. The drive power level $P_{c}$ is chosen so that the amplitude of motion $a_{1}$ in Eq. (18) is equal to the beam thickness $t$, approximately the amplitude for the onset of nonlinearity. The calculated temperature rise due to dissipation in the resonator from this level of drive power is very small, of order $0.1 \mathrm{~K}$. We also display for comparison the Allan variance for an oven-controlled $10 \mathrm{MHz}$ quartz crystal oscillator, the HP10811D. ${ }^{22}$ For quality factors higher than $10^{5}$, the calculated thermally induced fluctuations are comparable to or better than those of the bulk quartz resonator.

\section{TEMPERATURE FLUCTUATIONS}

We now turn to a discussion of the effects of finite thermal conductance and heat capacitance on the Allan variance. The small dimensions associated with our model resonator, and of nanoscale resonators in general, imply that the heat capacity of the resonator is very small. The corresponding thermal fluctuations are proportionally larger, and these may in turn produce significant frequency fluctuations, due to the temperature dependence of the resonator material parameters and geometric dimensions. Here, we present a simple model through which the magnitude of these effects can be estimated.

A heat capacitance $c$, connected by a thermal conductance $g$ to an infinite thermal reservoir at temperature $T$, will have an average thermal energy $\left\langle E_{c}\right\rangle=c T$ in the absence of any power loads. Changes in the temperature relax with the 


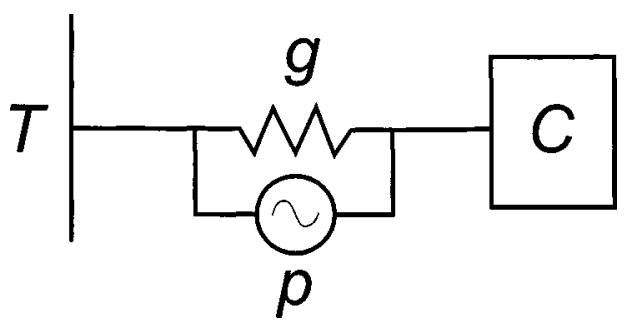

FIG. 5. Thermal circuit with a finite thermal conductance $g$ and a finite heat capacitance $c$, including a power noise source $p$.

thermal time constant $\tau_{T}=c / g$. The energy $E_{c}$ will however fluctuate, as the fluctuation-dissipation theorem applies to finite thermal conductances in a manner similar to the dissipation-induced mechanical noise. ${ }^{23}$ The thermal circuit therefore includes a power noise source $p$ with spectral density $S_{p}(\omega)=2 k_{B} T^{2} g / \pi$ (see Fig. 5). The instantaneous energy of the heat capacitance can then be written $E_{c}(t)=c T$ $+\delta E(t)$, where the spectral density of the energy fluctuations $\delta E(t)$ can be derived from the thermal circuit,

$$
S_{E}(\omega)=\frac{2}{\pi} \frac{k_{B} T^{2} c^{2} / g}{1+\omega^{2} \tau_{T}^{2}}
$$

We can interpret the energy fluctuations as temperature fluctuations $\delta T_{c}(t)$, if we define the temperature as $T_{c}=E_{c} / c$. The corresponding spectral density of the temperature fluctuations is given by

$$
S_{T}(\omega)=\frac{2}{\pi} \frac{k_{B} T^{2} / g}{1+\omega^{2} \tau_{T}^{2}} .
$$

At low frequencies $\omega$, below that of the thermal frequency $1 / \tau_{T}$, the temperature fluctuations $\delta T$ follow those driven by the noise source $p$, while at higher frequencies the nonzero heat capacitance acts as a filter.

For a resonator with the geometry shown in Fig. 1, there is no clear separation of the structure into a distinct heat capacitance and thermal conductance. Instead, we divide the resonator into slices of length $\Delta x$ and cross-sectional area $A=w \times t$, so that the $n$th slice has heat capacity $c_{n}$ $=C_{v} A \Delta x$, where $C_{v}$ is the specific heat per unit volume. The $(n-1)$ th and $n$th slices are connected to one another by the thermal conductance $g_{n}=\kappa A / \Delta x$, with thermal conductivity $\kappa$ given by the classical formula, $\kappa=(1 / 3) C_{V} c_{s} \ell(\ell$ is the phonon mean-free path and $c_{s}$ the sound speed). The thermal conductances $g_{n}$ are associated with noise power sources $p_{n}$, with spectral density $S_{p_{n}}(\omega)=2 k_{B} T^{2} g_{n} / \pi$. Finally, the temperatures at the ends of the beam, where the beam is mechanically clamped, are assumed to be given by the reservoir temperature $T$; see Fig. 6 .

In this model, energy fluctuations in the slices $n-1$ and $n$ are anticorrelated through the shared conductance $g_{n}$ : An energy $\delta E$ driven into the $n$th slice by $p_{n}$ corresponds to the same energy taken from the $(n-1)$ th slice. These energy fluctuations then relax through conductance into adjacent slices, and so on through the beam length, so that there is some correlation between the fluctuations in all slices, although the correlations get weak for distant slices.

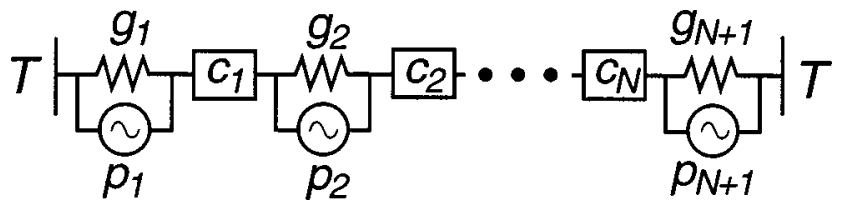

FIG. 6. Thermal model for doubly clamped beam, consisting of a series connection of heat capacitances $c_{n}$ and thermal conductances $g_{n}$, each associated with a cross-sectional slice of the beam of length $\Delta x$. Each thermal conductance is associated with a power noise source $p_{n}$. The ends are assumed clamped at the reservoir temperature $T$. There are a total of $N$ $=L / \Delta x$ elements.

One might expect that the most accurate model would use slices with differential lengths $\Delta x=d x \rightarrow 0$. However, once the slices become shorter than the phonon mean-free path $\ell$, the temperature in a slice is no longer well defined. We therefore choose slices with a length $\Delta x=\ell=50 \mathrm{~nm}$, so that each element has a volume $V=50 \times 50 \times 50 \mathrm{~nm}^{3}$. The corresponding heat capacity is $c=C_{V} V=2 \times 10^{-16} \mathrm{~J} / \mathrm{K}$, and the thermal links have $g=\kappa \ell=7.4 \times 10^{-6} \mathrm{~W} / \mathrm{K}$. The thermal time constant is $\tau_{T}=30 \mathrm{ps}$, corresponding to thermal frequencies $\sim 35 \mathrm{GHz}$, well outside the range of frequencies of interest for resonator phase fluctuations, $\Omega_{1} / Q \ll \omega$ $\ll \Omega_{1}$. For the purposes of this calculation, therefore, we can treat the thermal fluctuations in the low-frequency limit.

Consider only the $n$th power source of the conductance $p_{n}$. If we take the frequency component at $\omega$, the $(n-1)$ th and $n$th slices have temperature variations $T_{n-1}(\omega)$ and $T_{n}(\omega)$ given by

$$
\begin{gathered}
(2+i \omega \tau) T_{n-1}=-\frac{p_{n}}{g}+T_{n-2}+T_{n} \\
(2+i \omega \tau) T_{n}=\frac{p_{n}}{g}+T_{n-1}+T_{n+1} .
\end{gathered}
$$

The corresponding equation for the $(n+m)$ th slice is given by

$$
\left(2+i \omega \tau_{T}\right) T_{n+m}=T_{n+m-1}+T_{n+m+1} .
$$

Taking the limit $\omega \tau_{T} \ll 1$, we find that the power source $p_{n}(\omega)$ driving the $n$th slice generates a temperature variation $T(\omega)=p_{n}(\omega) / 2 g$ uniformly across the beam. The corresponding anticorrelated source $-p_{n}(\omega)$ driving the $(n-1)$ th slice generates an equal but opposite temperature variation. Hence, in the limit $\omega \tau_{T} \ll 1$, the fluctuations driven by conductances within the beam have no net effect.

The other source of temperature fluctuations comes from the conductances at the beam ends, $g_{1}$ and $g_{N+1}$. These also drive the beam uniformly, but as the energy that appears in the first and last elements does not have an adjacent anticorrelated source, there is now a net effect. The final result from this model is that the temperature of all the elements in the beam fluctuate uniformly, with spectral density $S_{T}(\omega)$ given by the incoherent sum of the two end sources,

$$
S_{T}(\omega)=\frac{4}{\pi} \frac{k_{B} T^{2} / g}{1+\omega^{2} \tau_{T}^{2}} \quad\left(\omega \tau_{T} \ll 1\right) .
$$


We have kept the frequency dependence of the thermal circuit in order to retain the high-frequency cutoff in the fluctuations.

The effect these temperature fluctuations have on the resonator behavior involves changes in the density and elastic modulus, which affect the frequency directly, and through changes in the overall resonator length. The density and elastic modulus determine the resonator frequency through the combination $\sqrt{E / \rho}$, which is the sound speed $c_{s}$; this quantity has the fractional temperature dependence $\left(1 / c_{s}\right) \partial c_{s} / \partial T=-5 \times 10^{-5} / \mathrm{K}$ for pure $\mathrm{Si}^{24}$ The corresponding temperature dependence of the resonator frequency is given by

$$
\frac{1}{\Omega_{n}} \frac{\partial \Omega_{n}}{\partial T}=\frac{1}{c_{s}} \frac{\partial c_{s}}{\partial T} \quad \text { (sound speed dependence). }
$$

The changes in resonator length generate longitudinal stress in the resonator, as the ends are assumed rigidly clamped. The resonator length $L$ changes with temperature due to the thermal expansion of $\mathrm{Si}$, with $(1 / L) \partial L / \partial T=\alpha_{T}$ $=2.8 \times 10^{-6} / \mathrm{K}$. A temperature change $\Delta T$ therefore induces a longitudinal extensional stress $\tau=-E \alpha_{T} \Delta T$. This in turn causes a change in the $n$th resonator frequency $\Omega_{n}(\tau)$, which for small extensional stress $\tau$ is given by ${ }^{15}$

$$
\Omega_{n}^{2}(\tau)=\Omega_{n}^{2}(0)+k_{n}^{2} \frac{\tau}{\rho}
$$

in terms of the beam eigenvectors $k_{n}$ and eigenfrequencies $\Omega_{n}=\sqrt{E I / \rho A} k_{n}^{2}$. The fractional frequency dependence due to length change is therefore

$$
\frac{1}{\Omega_{n}} \frac{\partial \Omega_{n}}{\partial T}=-\frac{1}{2} \frac{E}{\rho} \frac{k_{n}^{2}}{\Omega_{n}^{2}} \alpha_{T} \quad \text { (length dependence). }
$$

Hence, the spectral density of fractional frequency fluctuations $S_{y}(\omega)$ caused by the combined temperature dependence on sound speed and beam length is given by

$$
\begin{aligned}
S_{y}(\omega) & =\left(\frac{1}{\Omega} \frac{\partial \Omega}{\partial T}\right)^{2} S_{T}(\omega) \\
& =\left(-\frac{c_{s}^{2} k_{n}^{2}}{\Omega_{n}^{2}} \alpha_{T}+\frac{2}{c_{s}} \frac{\partial c_{s}}{\partial T}\right)^{2} \frac{k_{B} T^{2} / \pi g}{1+\omega^{2} \tau_{T}^{2}} .
\end{aligned}
$$

For the fundamental mode, we have $k_{1}=4.73 / L$, and inserting the model resonator parameters, we find

$$
S_{y}(\omega)=\left(1.6 \times 10^{-8} / \mathrm{K}^{2}\right) \frac{k_{B} T^{2} / g}{1+\omega^{2} \tau_{T}^{2}}=\frac{2.7 \times 10^{-21}}{1+\omega^{2} \tau_{T}^{2}} \frac{1}{\mathrm{rad} / \mathrm{s}} .
$$

From the fractional frequency noise, Eq. (60), we calculate the Allan variance:

$$
\begin{aligned}
\sigma_{A}\left(\tau_{A}\right) & =\left(2.25 \times 10^{-4} / \mathrm{K}^{2}\right) \sqrt{k_{B} T^{2} / g \tau_{A}} \\
& =9.3 \times 10^{-11} \frac{1}{\sqrt{\tau_{A}}},
\end{aligned}
$$

in the limit $\tau_{A} \gg \tau_{T}$. The contribution of thermal fluctuations to the Allan variance for our model resonator is therefore of the same magnitude and dependence on averaging time $\tau_{A}$ as that due to mechanical dissipation for a resonator with $Q$ of about $10^{4}$, and is a significant source of fluctuation. Ways to reduce the size of this source of variance include the use of materials with larger thermal conductance, such as AlN (3.20 $\mathrm{W} / \mathrm{cm} \mathrm{K})^{25}$ and sapphire $(4.50 \mathrm{~W} / \mathrm{cm} \mathrm{K})$, or better temperature-compensated materials, such as quartz.

\section{OTHER SOURCES OF NOISE}

There are a number of other sources of noise that can affect resonator performance. We discuss here two such sources, one due to adsorption-desorption noise of residual gas molecules, and the other due to defect motion within the resonator structure.

\section{A. Adsorption-desorption noise}

Adsorption-desorption noise has been discussed in some detail by Vig and $\mathrm{Kim}^{12}$ and Yong and Vig. ${ }^{11}$ The resonator environment will always include a nonzero pressure of surface-contaminating molecules. These molecules, when they adsorb on a site on the resonator surface, mass load the resonator, and thereby change its resonance frequency. As the molecules adsorb and desorb due to their finite binding energy and nonzero temperature, the resulting changes in frequency translate to a source of phase or fractional frequency noise. As discussed herein, this type of noise does not fit into the Zener formalism, as the adsorptiondesorption cycle is not intrinsically a dissipative one: As the arrival and departure times of the atoms are random, they do not on average change the energy of the resonator, but cause its frequency to change in a discontinuous fashion, leaving the quality factor unchanged. This type of parametric noise (where the overall resonator mass is fluctuating) is therefore not described by the lossy stress-strain relation developed by Zener.

The frequency change due to a single adsorbed molecule, $\Delta \Omega$, is proportional to the ratio of the molecule to resonator total mass, $m / M$; smaller mass resonators are more sensitive than larger ones. Furthermore, as the resonator size scale is reduced, the number of adsorption sites $N_{a}$ on the resonator surface grows in proportion to the number of total number of resonator atoms: The surface-to-volume ratio grows inversely to the size scale. Hence, nanoscale resonators are more susceptible to adsorption-desorption noise than larger, bulk mechanical resonators.

We use a simple model to estimate the noise from this source. We assume a single molecular species with mass $m$, surface binding energy $E_{b}$, and pressure $P$. Expressions may be derived for the adsorption and desorption rates $r_{a}$ and $r_{d}$ at any given surface site; ${ }^{11}$ with sticking coefficient $s$, the adsorption rate at any site is given by

$$
r_{a}=\frac{2}{5} \frac{P}{\sqrt{m k_{B} T}} s .
$$

The sticking coefficient is typically temperature dependent. ${ }^{26}$ Once bound to the surface, a molecule desorbs at a rate 


$$
r_{d}=\nu_{d} \exp \left(-\frac{E_{b}}{k_{B} T}\right)
$$

where $\nu_{d}$ is the desorption attempt frequency, typically of order $10^{13} \mathrm{~Hz}$, and $E_{b}$ is the desorption energy barrier. The average occupation $f$ of a site is then $f=r_{a} /\left(r_{a}+r_{d}\right)$; the variance in the occupation probability is $\sigma_{\mathrm{occ}}^{2}=r_{a} r_{d} /\left(r_{a}\right.$ $\left.+r_{d}\right)^{2}$.

An expression may then be derived for the phase noise $S_{\phi}(\omega)$ resulting from the statistics of the adsorptiondesorption process: ${ }^{11}$

$$
S_{\phi}(\omega)=\frac{2 N_{a} \sigma_{\mathrm{occ}}^{2} \tau_{A} / \pi}{1+\omega^{2} \tau_{r}^{2}} \frac{\Delta \Omega^{2}}{\omega^{2}} .
$$

Here, $\tau_{r}$ is the correlation time for an adsorption-desorption cycle, $1 / \tau_{r}=r_{a}+r_{d}$. A simple mass-loading formula may be assumed, $\Delta \Omega=(\mathrm{m} / 2 M) \Omega_{1}$, where $M$ is the resonator mass and $\Omega_{1}$ is the fundamental frequency of the resonator. A corresponding expression for the fractional frequency noise is then given by

$$
S_{y}(\omega)=\frac{N_{a} \sigma_{\mathrm{occ}}^{2} \tau_{r} / 2 \pi}{1+\omega^{2} \tau_{r}^{2}}\left(\frac{m}{M}\right)^{2} .
$$

We calculate the Allan variance,

$$
\sigma_{A}\left(\tau_{A}\right)=\sqrt{\frac{N_{a} \tau_{r}}{2 \tau_{A}}} \frac{\sigma_{\mathrm{occ}} m}{M}
$$

in the limit $\tau_{A} \gg \tau_{r}$.

The occupation variance $\sigma_{\text {occ }}^{2}$, and therefore the noise, is maximum when the site occupation probability is $f=0.5$, i.e., when the adsorption and desorption rates are equal. The noise is minimized when the occupation probability is either near zero or near unity. For typical packaged pressures, molecular sticking coefficients, and binding energies, occupation probabilities are quite small; we therefore try to minimize the occupation to reduce the fluctuation variance. The exponential dependence of the desorption rate $r_{d}$ on temperature provides a useful approach; heating the resonator, using an on-chip heating element, causes significant increases in the desorption rate and therefore in the occupation variance $\sigma_{\text {occ }}^{2}$.

In Fig. 7, we plot the Allan variance for a $1 \mathrm{~s}$ averaging interval, as a function of package pressure, for two resonator temperatures, 300 and $500 \mathrm{~K}$. We have chosen a contaminant molecule with an binding energy of $E_{b}=10 \mathrm{kcal} / \mathrm{mol}$, with one adsorption site every $0.25 \mathrm{~nm}^{2}$, and a sticking coefficient of 0.1 , typical values for gas molecules adsorbing on metal surfaces. ${ }^{11,26}$ Note that the sticking coefficient is typically temperature dependent, ${ }^{26}$ but here we have taken it as constant. We show for comparison the overall Allan variance for the HP 10811D $10 \mathrm{MHz}$ quartz oscillator, again for a $1 \mathrm{~s}$ averaging interval.

It is clear from Fig. 7 that this source of noise is extremely important. Great care must be taken to passivate the resonator surface, thus reducing the sticking coefficient, reduce the pressure, for instance by including getters in the package, and possibly raising the ambient temperature.

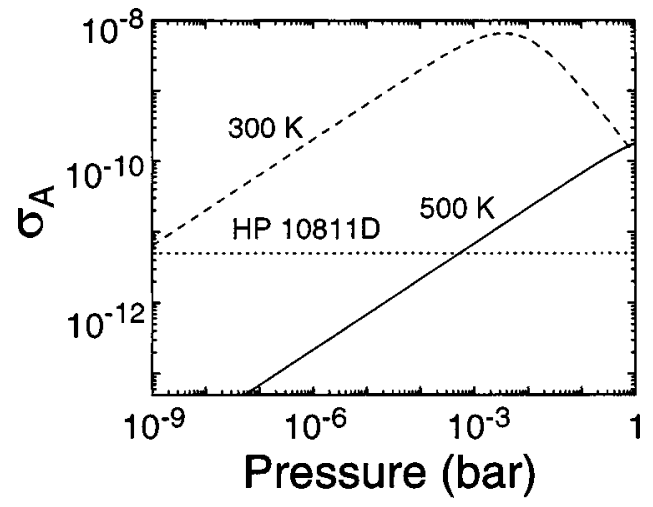

FIG. 7. Allan variance for a $1 \mathrm{~s}$ averaging interval as a function of package pressure for two resonator temperatures, 300 and $500 \mathrm{~K}$. The contaminant molecule has a binding energy $E_{b}=10 \mathrm{kcal} / \mathrm{mol}$, with one adsorption site every $0.25 \mathrm{~nm}^{2}$, and a sticking coefficient of 0.1 . Also shown is the fractional noise for the HP10811D $10 \mathrm{MHz}$ quartz resonator, for a $1 \mathrm{~s}$ averaging interval.

\section{B. Defect motion}

The last source of noise we would like to consider is that caused by defect motion within the resonator volume. For the single-crystal resonators we have been considering, defect levels are very low in the base material. Statistically, there are of order 0 to 1 defects within the volume of the resonator. We therefore do not expect this to provide a serious source of noise in these resonators.

However, there has been extensive work on developing polycrystalline materials for resonators, especially using silicon nitride and poly-Si. ${ }^{5,6}$ These materials include a large density of grain boundaries, point defects, and some voids, and the motion of these defects can cause phase and frequency noise in high- $Q$ resonators. Amorphous $\mathrm{Si}$ created by implantation has defect densities of order $1 \% ;{ }^{27}$ similar levels are expected for chemical vapor deposition-grown polysilicon. The quality factors of resonators fabricated from this material are comparable, at room temperature, to those made from single-crystal materials such $\mathrm{Si}$ and $\mathrm{GaAs}$, but defect processes may still play a very important role.

Point defects in a solid can be treated as elastic dipoles, with symmetry different from that of the underlying crystal (see e.g., Nowick and Berry ${ }^{13}$ ). In the relaxed state, the defects are randomly oriented; reorientations occur due to thermally induced motion, at a rate $\Gamma_{d}=\nu_{0} \exp \left(-\Delta g^{*} / k_{B} T\right)$, with an attempt frequency $\nu_{0} \sim 10^{12} \mathrm{~Hz}$ and free energy barrier $\Delta g^{*}$, typically of order $0.1-1 \mathrm{eV}$. If we consider a defect with two possible orientations, with equal energies, the occupation probabilities are given by the Gibbs distribution and are each equal to $1 / 2$; the variance in the mean occupation is equal to $1 / 4$, with mean reorientation time $\tau_{d}=1 / \Gamma_{d}$. Defects in which the possible orientations have different energies will have smaller variances in the occupation, and there will be a range of reorientation times as well.

A single defect moving or reorienting itself can cause a change in the local Young's modulus $E$; if the defect has two possible configurations, - and + , the corresponding local modulus changes from $E_{s}+E_{-}$to $E_{s}+E_{+}$, where $E_{s}$ is the 
TABLE IV. Expressions for the various spectral noise densities worked out for different representations of the same noise, as well as for noise associated with different fundamental processes.

\begin{tabular}{|c|c|c|c|}
\hline Type of noise & Symbol & Expression & Equation \\
\hline Force noise, mode $n$ & $S_{f_{n}}(\omega)$ & $\frac{2 k_{B} T M \Omega_{n}}{\pi Q L^{2}}$ & (23) \\
\hline \multirow{2}{*}{ Amplitude noise, mode $n$} & \multirow{2}{*}{$S_{a_{n}}(\omega)$} & $\Omega_{n} \quad 2 k_{B} T$ & \multirow{2}{*}{ (24) } \\
\hline & & $\overline{\left(\Omega_{n}^{2}-\omega^{2}\right)^{2}+\left(\Omega_{n}^{2} / Q\right)^{2}} \overline{\pi M L^{2} Q}$ & \\
\hline On-resonance amplitude noise, mode $n$ & $S_{a_{n}}\left(\Omega_{n}\right)$ & $\frac{2 k_{B} T Q}{\pi M L^{2} \Omega_{n}}$ & (24) \\
\hline Off-resonance phase noise, mode $n=1$ & $S_{\phi}(\omega)$ & $\frac{k_{B} T}{8 \pi P_{c} Q^{2}}\left(\frac{\Omega_{1}}{\omega}\right)^{2}$ & (34) \\
\hline Off-resonance fractional frequency noise, $n=1$ & $S_{y}(\omega)$ & $\frac{k_{B} T}{8 \pi P_{c} Q^{2}}$ & (42) \\
\hline Allan variance, $n=1$ & $\sigma_{A}\left(\tau_{A}\right)$ & $\sqrt{\frac{k_{B} T}{8 P_{c} Q^{2} \tau_{A}}}$ & (49) \\
\hline Temperature fluctuations & $S_{T}(\omega)$ & $\frac{4}{\pi} \frac{k_{B} T^{2} / g}{1+\omega^{2} \tau_{T}^{2}}$ & (55) \\
\hline Allan variance, temperature fluctuations & $\sigma_{A}\left(\tau_{A}\right)$ & $\left(2.25 \times 10^{-4} / \mathrm{K}^{2}\right) \sqrt{k_{B} T^{2} / g \tau_{A}}$ & (61) \\
\hline Allan variance, adsorption desorption & $\sigma_{A}\left(\tau_{A}\right)$ & $\sqrt{\frac{2 N_{a} \tau_{r}}{\tau_{A}}} \frac{\sigma_{\mathrm{occ}} m}{M}$ & (66) \\
\hline Allan variance, defect motion & $\sigma_{A}\left(\tau_{A}\right)$ & $\sqrt{\frac{2 \sigma_{\omega}^{2}}{\langle\Omega\rangle^{2}}} \sqrt{\frac{\tau_{d}}{\tau_{A}}}$ & (68) \\
\hline
\end{tabular}

defect-free modulus. Typical values for $E_{ \pm}$are in the range $0.01-0.1 E_{s}$. A mole fraction $C_{d} \ll 1$ of such defects, all simultaneously reorienting from - to + , will cause the effective modulus to change ${ }^{13}$ from $E_{s}+C_{d} E_{-}$to $E_{s}+C_{d} E_{+}$. If we consider a total mole fraction of identical defects $C_{0}$ $\ll 1$, that reorient independently between configurations - and + with equal free energies, so that the two configurations are equally likely, the average elastic modulus for the solid will be $\langle E\rangle \approx E_{s}+C_{0}\left(E_{-}+E_{+}\right) / 2$. The variance in the elastic modulus is given by the Poisson formula, $\sigma_{E}^{2}$ $\approx C_{0}\left(E_{+}-E_{-}\right)^{2} / 4$. Additional noise sources can appear from the defect motion itself; the defect may resonate at a frequency near the resonator natural frequency, causing additional dissipation and additional noise terms. We ignore such effects here.

Applying this discussion to resonator frequency fluctuations, if we assume the defects all have equal impact on the resonator frequency $\Omega$, the mean resonator frequency $\langle\Omega\rangle$ will be that calculated from Eq. (3) using the average modulus $\langle E\rangle$. The frequency will have a mean variance given by $\sigma_{\Omega}^{2}=\left(C_{0} / 8\right)\left(\Omega_{+}-\Omega_{-}\right)^{2}$, where $\Omega_{ \pm}$are calculated from Eq. (3) using the moduli $E_{s}+C_{0} E_{ \pm}$.

These fluctuations, occurring with a single reorientation time $\tau_{d}$, will generate fractional frequency noise with the spectral density

$$
S_{y}(\omega)=\frac{2}{\pi} \frac{\sigma_{\Omega}^{2}}{\langle\Omega\rangle^{2}} \frac{\tau_{d}}{1+\omega^{2} \tau_{d}^{2}} .
$$

For this distribution, the corresponding Allan variance has a similar functional form to that shown in Fig. 3. The defect reconfiguration time $\tau_{d}$ can range from $\mu$ s to minutes or hours at room temperature, with an exponential temperature dependence. For averaging times $\tau_{A}$ much larger than $\tau_{d}$, the approximate form for the Allan variance is

$$
\sigma_{A}\left(\tau_{A}\right)=\sqrt{\frac{2 \sigma_{\omega}^{2}}{\langle\Omega\rangle^{2}}} \sqrt{\frac{\tau_{d}}{\tau_{A}}} .
$$

If we assume a defect mole fraction $C_{0}=0.001$, and modulus changes $E_{ \pm}= \pm 0.1 E_{s}$, the frequency variance is $\sigma_{\Omega} \approx 1$ $\times 10^{-6} \Omega$. For a defect reconfiguration time $\tau_{d}=1 \mathrm{~ms}$, we find an Allan variance at $\tau_{A}=1 \mathrm{~s}$ of $5 \times 10^{-8}$, a quite large contribution compared to those we have been considering. Clearly, active defect concentrations $C_{0}$ of less than 1 part in $10^{5}$ are needed to achieve Allan variances competitive with those of single-crystal resonators.

We note that a typical solid will include a range of reorientation times $\tau_{d}$, which when superposed generically produce $1 / f$ noise through the Dutta-Dimon-Horn model. ${ }^{28}$ In that case, we can write

$$
S_{y}(\omega) \approx A \frac{\omega_{c}}{\omega} k_{B} T \mathcal{D}(\bar{E})
$$

where $\mathcal{D}(\bar{E})$ is the density of defect states at energy $\bar{E}$ $=-k_{B} T \log _{e}\left(\omega / \nu_{0}\right)$ and $A$ is a scale factor. In this case the Allan variance works out to be [see Eq. (46)] 


$$
\sigma_{A}\left(\tau_{A}\right) \approx \sqrt{2 \log _{e} 2 A \omega_{c}} \sqrt{k_{B} T \mathcal{D}(\bar{E})} .
$$

Here, we see the Allan variance independent of averaging time $\tau_{A}$, but with a magnitude comparable to that of Eq. (68).

Such processes, which play an important role in singlecrystal and polycrystalline metals, and for which much information in metals exists, need to be investigated in silicon nitride and poly-Si to determine whether these play an important role in limiting resonator performance.

\section{CONCLUSIONS}

We have described a formalism for treating the resonance behavior, loss processes, and resulting frequency and phase noise in nanoscale resonators. We then applied this formalism to evaluate the role of a number of fundamental and material-dependent noise sources, and how these sources affect the frequency stability of a model $1 \mathrm{GHz}$ nanomechanical resonator. For practical applications, the relevant comparison has been made with an industry standard, the oven-controlled high-precision quartz crystal. We find that the anticipated resonator noise is predominantly from thermomechanical noise, temperature fluctuations, and adsorption-desorption noise. The noise levels from these sources are comparable in magnitude to that of the quartz crystal, provided some care is taken to minimize certain important loss processes. In Table IV, we have tabulated the various expressions for the noise, from different treatments and from different noise sources.

The results we have calculated here are for a doubly clamped, flexural resonator. Any resonator whose basic equations of motion can be reduced to those of a linear simple harmonic oscillator, driven by a force term, will have results of the form shown here. The results for a cantilevered beam, a torsional resonator, and a longitudinal wave resonator will therefore all be identical to these, except that the resonance frequencies and the mode shapes are different, so that the numerical prefactors will be somewhat different.

For practical applications, nanoscale resonators can be fabricated on chip with electronics needed to provide precision frequency control. This would obviate the need for an externally packaged and controlled quartz crystal, and enable integrated fabrication.

Clearly, there are gaps in the available data for evaluating whether the noise performance calculated here can be achieved in fact. More systematic approaches, measuring the performance of high- $Q$ resonators operated in phase-locked loops, with controlled variations in temperature, environment, and materials, need to be followed in order to deter- mine whether the suggested applications are indeed viable. A better understanding of the role of defects and molecular adsorption and desorption is also needed to evaluate the effect these have on frequency stability.

\section{ACKNOWLEDGMENTS}

The authors acknowledge the financial support provided by the National Science Foundation XYZ-On-A-Chip Program, Contract No. ECS-9980734, by the Army Research Office, and by the Research Corporation through a Research Innovation Award.

${ }^{1}$ A. N. Cleland and M. L. Roukes, Appl. Phys. Lett. 69, 2653 (1996).

${ }^{2}$ A. N. Cleland and M. L. Roukes, Nature (London) 320, 160 (1998).

${ }^{3}$ Y. T. Yang, K. L. Ekinci, X. M. H. Huang, L. M. Schiavone, M. L. Roukes, C. A. Zorman, and M. Mehregany, Appl. Phys. Lett. 78, 162 (2001).

${ }^{4}$ K. Yasumura, T. Stowe, E. Chow, T. Pfafman, T. Keeny, B. Stipe, and D. Rugar, J. Microelectromech. Syst. 9, 117 (2000).

${ }^{5}$ C. Nguyen and R. Howe, Proc. IEEE Intl. Freq. Control Symp. 48, 127 (1994).

${ }^{6}$ K. Wang, A. Wong, and C. Nguyen, J. Microelectromech. Syst. 9, 347 (2000).

${ }^{7}$ R. Lifshitz and M. Roukes, Phys. Rev. B 61, 5600 (2000).

${ }^{8}$ F. Walls and J. Vig, IEEE Trans. Ultrason. Ferroelectr. Freq. Control 42, 576 (1995).

${ }^{9}$ T. Gabrielson, IEEE Trans. Electron Devices 40, 903 (1993).

${ }^{10}$ L. Cutler and C. Searle, Proc. IEEE 54, 136 (1966).

${ }^{11}$ Y. Yong and J. Vig, IEEE Trans. Ultrason. Ferroelectr. Freq. Control 36, 452 (1989).

${ }^{12} \mathrm{~J}$. Vig and Y. Kim, IEEE Trans. Ultrason. Ferroelectr. Freq. Control 46, 1558 (1999)

${ }^{13}$ A. Nowick and B. Berry, Anelastic Relaxation in Crystalline Solids (Academic, New York, 1972).

${ }^{14}$ T. Albrecht, P. Grütter, D. Horne, and D. Rugar, J. Appl. Phys. 69, 668 (1991)

${ }^{15}$ S. Timoshenko, D. Young, and J. W. Weaver, Vibration Problems in Engineering (Wiley, New York, 1974).

${ }^{16}$ X. Huang, K. Ekinci, Y. Yang, C. Zorman, and M. L. Roukes, (unpublished).

${ }^{17}$ A. N. Cleland and M. L. Roukes, Sens. Actuators A 72, 256 (1999).

${ }^{18}$ W. Egan, IEEE Trans. Instrum. Meas. 37, 240 (1988).

${ }^{19}$ W. Robins, Phase Noise in Signal Sources (Peter Peregrinus Ltd., London, 1982).

${ }^{20}$ D. Allan, Proc. IEEE 54, 221 (1966).

${ }^{21}$ W. Egan, Frequency Synthesis by Phase Lock (Wiley, New York, 1981).

${ }^{22}$ Hewlett-Packard 10811D/E application note.

${ }^{23}$ L. Landau, E. Lifshitz, and L. Pitaevskii, Statistical Physics, 4th ed. (Pergamon, Oxford, 1980).

${ }^{24}$ W. Mason and T. Bateman, Phys. Rev. A 134, 1387 (1964).

${ }^{25}$ G. S. et al., J. Phys. Chem. Solids 48, 641 (1987).

${ }^{26}$ A. Zangwill, Physics at Surfaces (Cambridge University Press, New York, 1988).

${ }^{27}$ S. Roorda, W. C. Sinke, J. M. Poate, D. C. Jacobson, D. Dierker, B. S. Dennis, D. J. Eaglesham, F. Spaepen and P. Fuoss, Phys. Rev. B 44, 3702 (1991).

${ }^{28}$ P. Dutta, P. Dimon, and P. Horn, Phys. Rev. Lett. 43, 646 (1979). 